\title{
Diagnóstico de helmintiasis por videocápsula endoscópica
}

\section{Diagnosis of helminthiasis by endoscopic videocapsule}

\author{
Lázaro A. Arango Molano, MD, ${ }^{1}$ Mario Jaramillo, MD, ${ }^{2}$ Julián Ferreira, MD, ${ }^{3}$ Johan Carvajal, MD, ${ }^{4}$ Marcos Cardozo, MD5
}

\footnotetext{
1 Gastroenterólogo Clínico Quirúrgico. Coordinador, Programa de Posgrado en Gastroenterología ClínicoQuirúrgica, Universidad de Caldas, Manizales, Colombia.

2 Medicina Interna. Gastroenterología. Docente, Universidad de Caldas, Manizales, Colombia.

3 Gastroenterólogo Clínico Quirúrgico, Universidad de Caldas, Manizales, Colombia.

${ }^{4}$ Gastroenterólogo Clínico Quirúrgico, Universidad de Caldas, Manizales, Colombia.

5 Fellow de Gastroenterología Clínico-Quirúrgica, Universidad de Caldas, Manizales, Colombia.

Correspondencia: Marcos Cardozo:

Marcosh83@hotmail.com

Fecha recibido: $14-08-17$ Fecha aceptado: $30-10-17$
}

\begin{abstract}
Resumen
La helmintiasis corresponde a una parasitosis intestinal altamente prevalente en países en desarrollo, y que viene aumentando en los países desarrollados. Teniendo en cuenta el ciclo de vida de los helmintos, su presentación se relaciona con situaciones ambientales y socioeconómicas de alto riesgo. Por tanto, su presencia endémica puede considerarse un problema de salud pública. Para el diagnóstico, se disponen de varios elementos que incluyen el estudio de la materia fecal, la endoscopia y las imágenes diagnósticas como los estudios de rayos $X$ de vías digestivas, la tomografía axial computarizada (TAC) y la resonancia magnética (RM). En la actualidad, existen reportes de diagnóstico de helmintiasis por cápsula endoscópica que también han permitido el estudio de la ecología y de la patología in situ de estos parásitos. Se presenta un caso clínico de helmintiasis compatible con estrongiloidosis, diagnosticado por cápsula endoscópica por el Grupo de Gastroenterología Clínico-Quirúrgica de la Universidad de Caldas, Colombia.
\end{abstract}

\section{Palabras clave \\ Strongyloides stercoralis, helmintiasis, endoscopía capsular.}

\begin{abstract}
Helminthiasis is an intestinal parasitosis that is very prevalent in developing countries but is increasing in developed countries. Taking into account the life cycle of helminths, their presentation is related to high-risk environmental and socioeconomic situations. Endemic presence can be considered a public health problem. Several methods are available for diagnosis including the study of fecal matter, endoscopy and diagnostic imaging such as X-ray studies of the digestive tract, CT scans and magnetic resonance. Recently, there have been reports of diagnosis of helminthiasis by capsule endoscopy which has also allowed the study of the ecology and in situ pathology of these parasites. We present a clinical case of helminthiasis compatible with strongyloidiasis that was diagnosed by endoscopic capsule by the surgical clinical gastroenterology group of the University of Caldas in Manizales, Colombia.
\end{abstract}

Keywords

Strongyloides stercoralis, helminthiasis, capsular endoscopy. Source: DeCS.

\section{INTRODUCCIÓN}

Los helmintos son organismos presentes en los países en vías de desarrollo, aquellos con pobres condiciones de salubridad, pero también en países no endémicos que cuentan con migrantes de áreas donde sí se presentan. Hacen parte de los agentes infecciosos de más amplia distribución en los humanos y atacan, sobre todo, a las regiones marginales, de bajos ingresos y con escasez de recursos básicos. En la actualidad, se estima que más de 1 millón de personas (en países 
en vías de desarrollo) están infectadas por lo menos por un tipo de helminto. Estos parásitos traen consigo morbilidad, dada por anemia ferropénica, convulsiones, hipertensión portal, diarrea crónica y dolor abdominal crónico. Es muy bien sabido que la morbilidad producida por estos parásitos lleva a un círculo vicioso de pobreza, poca productividad y desarrollo socioeconómico inadecuado $(1,2)$. También son responsables de los altos niveles de morbilidad y mortalidad, especialmente de anemia por deficiencia de hierro, convulsiones, hipertensión portal y diarrea crónica (3).

Los helmintos son gusanos multicelulares, que en su forma adulta no pueden multiplicarse en el cuerpo humano y se transmiten generalmente por el contacto con las heces infectadas y a través del suelo, la comida o el agua contaminados. Miden entre $1 \mathrm{~mm}$ y varios metros dependiendo de la especie y viven en el tracto gastrointestinal, ya sea en el intestino delgado o el colon, donde se nutren del contenido endoluminal o por succión de la sangre de la pared intestinal. En Colombia, su prevalencia no tiene datos recientes, pero la Encuesta Nacional de Parasitismo Intestinal de 1980 reportó un 33,6 \% para Ascaris lumbricoides, un 37,5\% para Trichuris trichiura y un $23 \%$ para Uncinaria sp. en la población general. En la Amazonía colombiana se demostró una alta prevalencia de geohelmintos en niños de 2 a 16 años, siendo el más frecuente el Strongyloides stercoralis, con un $49,3 \%(4,5)$. A causa de la globalización, los helmintos han aumentado su prevalencia en países desarrollados, como Estados Unidos, Japón y algunos países europeos $(6,7)$.

Para el diagnóstico de las parasitosis, los Centros para el Control y la Prevención de Enfermedades (CDC) han establecido las siguientes pruebas: examen fecal, endoscopia, pruebas serológicas y exámenes de imágenes, que incluyen rayos $\mathrm{X}$, resonancia magnética $(\mathrm{RM})$ y tomografía axial computarizada (TAC) (8).

La cápsula endoscópica fue reportada por primera vez en el año 2000, aprobada por la FDA en 2001 y se estableció como un método no invasivo para el estudio del intestino delgado. La indicación más importante para su uso actualmente es el sangrado digestivo de origen oscuro. Permite, además, la evaluación del intestino delgado en toda su extensión, con imágenes de alta resolución, sin requerir sedación, cirugía o exposición a radiación $(9,10)$. El sistema consta de tres elementos fundamentales: la cápsula, una grabadora de datos y una estación de trabajo. La cápsula contiene un domo óptico, lentes, fuente de luz, batería, transmisor y una antena. El ángulo de visión va de $140^{\circ} \mathrm{a}$ $170^{\circ}$, con $8 \mathrm{X}$ de magnificación.

A continuación, se presenta un caso de diagnóstico de helmintiasis por medio de cápsula endoscópica, tratado por el grupo de Gastroenterología Clínico-Quirúrgica de la Universidad de Caldas, Colombia.

\section{CASO CLÍNICO}

Varón de 18 años, con clínica de 2 años de evolución caracterizada por episodios intermitentes de dolor abdominal tipo cólico generalizado, algunas veces asociado con deposiciones diarreicas sin sangre. Dichos episodios resolvían de manera espontánea, y por tratarse de un paciente que frecuentaba áreas rurales del trópico colombiano, recibía tratamientos antiparasitarios empíricos que incluían albendazol, nitazoxanida y secnidazol. Como único antecedente de importancia, sufre de dislipidemia familiar, manifestada por hipertrigliceridemia, para lo que toma gemfibrozilo.

El paciente presentó exacerbación del cuadro de dolor abdominal, predominantemente epigástrico, esta vez asociado con deposiciones diarreicas, vómito y fiebre, por lo que requirió ingreso al servicio de urgencias de una institución de tercer nivel. Se le realizaron múltiples estudios a su ingreso, que incluían valores séricos de lipasa y amilasa normales; electrólitos séricos normales y ecografía abdominal total, dentro de límites normales. La endoscopia digestiva alta concluyó hallazgos de gastritis folicular antral. Se le realizaron estudios de coprológico y coproscópico, negativos para parásitos intestinales, no concluyentes para infección. El coprocultivo fue negativo. Se llevó a cabo una TAC de abdomen, que estuvo dentro de los límites normales. El cuadro hemático inicial reportó leucocitos de 17210 con neutrófilos del 30,9 \% y eosinófilos del 43,9\%, hemoglobina de $14,3 \mathrm{~g} / \mathrm{dL}$ y hematocrito del 48,1\%. Ante estos hallazgos de eosinofilia y leucocitosis, se decidió complementar el estudio con una prueba de gota gruesa, la cual fue negativa. Se realizó colonoscopia izquierda, sin evidencia de lesiones, con toma de muestras del colon sigmoides y del recto. El resultado de patología: colitis indeterminada, proctitis crónica. Se realizó nuevo cuadro hemático con leucocitos de 19350, neutrófilos del 24,9\%, eosinófilos del $59 \%$, hemoglobina de $14,5 \mathrm{~g} / \mathrm{dL}$ y hematocrito del $42,6 \%$. En ese momento fue valorado por hematología, que consideró llevar a cabo aspirado de la médula ósea, el cual reportó evidencia de eosinofilia con células con adecuada maduración y médula ósea hipercelular con hiperplasia eosinofílica. Su último cuadro hemático intrahospitalario reportó leucocitos de 17500 , con neutrófilos del $15 \%$, eosinófilos del $64,5 \%$, hemoglobina de $15 \mathrm{~g} / \mathrm{dL}$ y hematocrito del $43,8 \%$. Se consideró posibilidad de síndrome hipereosinofílico, aunque para dicho diagnóstico se requiere un órgano blanco aún no identificado. Como segunda posibilidad se consideró leucemia eosinofílica. El paciente logró un control adecuado del dolor abdominal y se dio egreso, para continuar controles y estudios ambulatorios por parte de hematología. Se indicó manejo con ciclo corto de esteroides y complementar el estudio con cápsula endoscópica 
y otros estudios hematológicos. El hallazgo de la cápsula endoscópica es concluyente, con evidencia de erosiones en el yeyuno (Figura 1) y una gran úlcera cubierta de fibrina a este nivel (Figura 2), donde posteriormente se aprecian múltiples parásitos intestinales pequeños, con una parte blanca y otra transparente, adheridos a la pared intestinal (Figuras 3 y 4). Se indicó manejo con Irvermectina 200 $\mathrm{mcg} / \mathrm{kg}$ dosis única repitiendo en 15 días, además de albendazol en $400 \mathrm{mg}$, por vía oral (VO), cada 12 horas, durante 10 días. El paciente logró control de sus episodios de dolor abdominal, y ahora está asintomático.

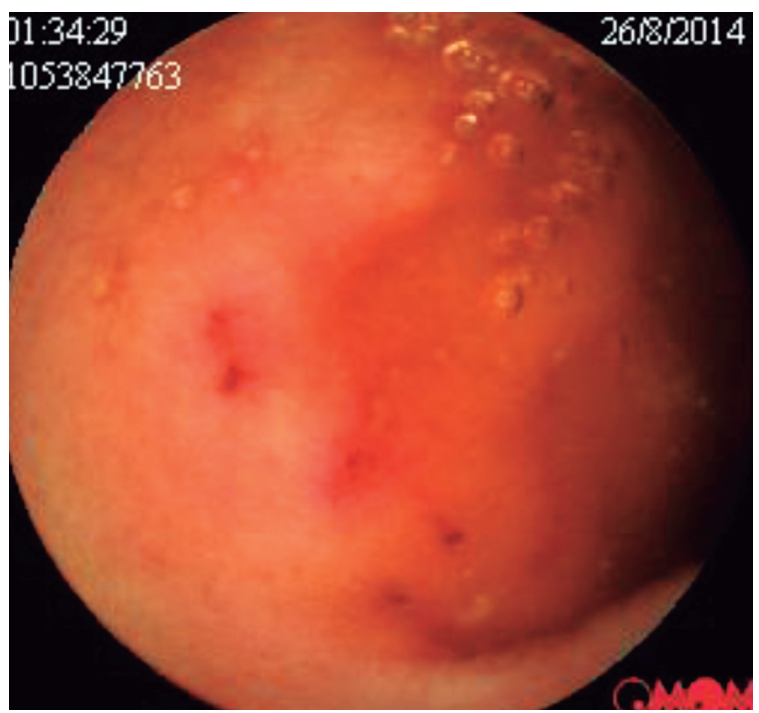

Figura 1. Erosiones a nivel yeyunal.

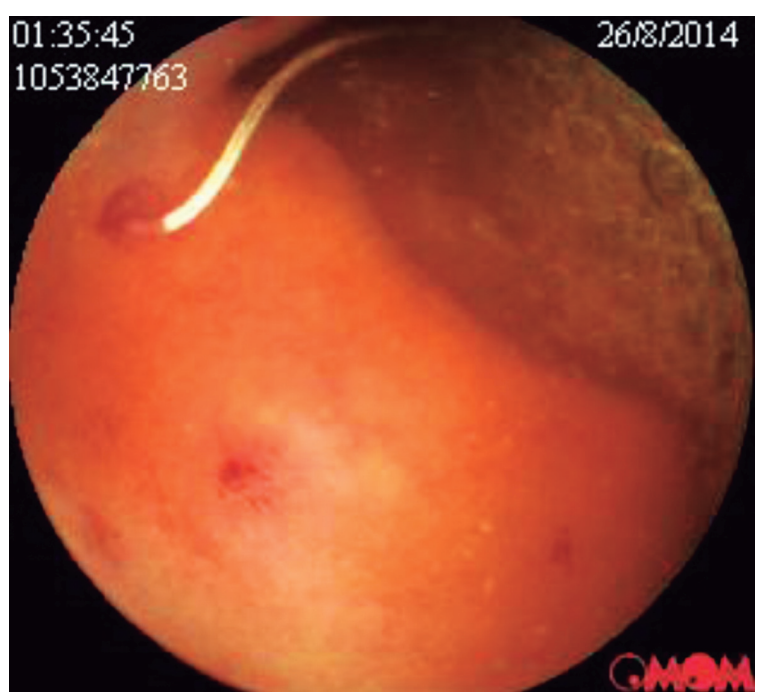

Figura 2. Parásitos intestinales blanquecinos adheridos a la pared intestinal.

\section{DISCUSIÓN}

A pesar de no ser uno de los métodos recomendados por los CDC para el diagnóstico de parásitos, la cápsula endoscópica puede ser utilizada para dicho fin. En el año 2003, Soares y colaboradores fueron los primeros en describir el uso de la cápsula endoscópica para la visualización in vivo de parásitos, en este caso, A. lumbricoides (11). La cápsula endoscópica puede ser utilizada no solo para evaluar la presencia, sino también la ecología y la patología en relación con la parasitosis. De igual manera, puede desempeñar un papel importante

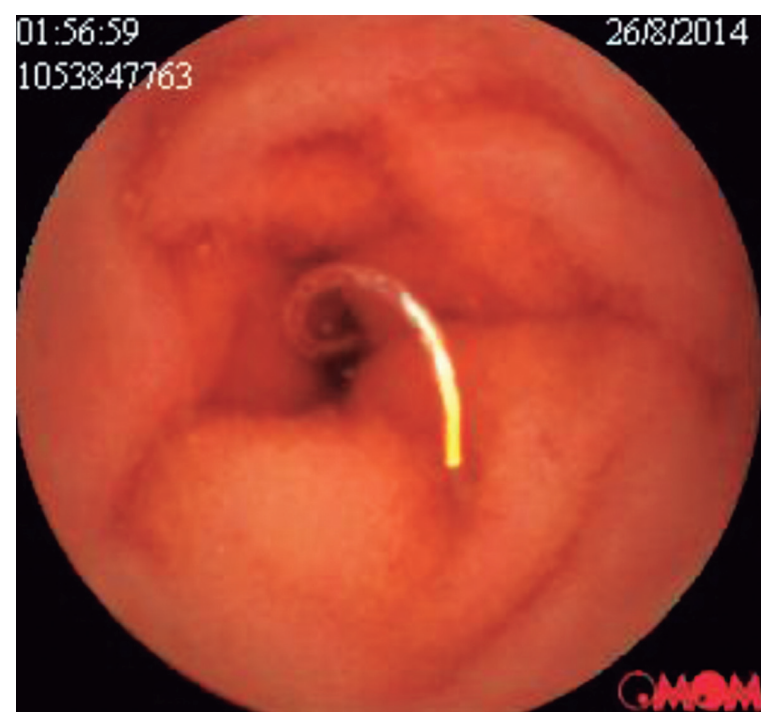

Figura 3. Parásito intestinal.

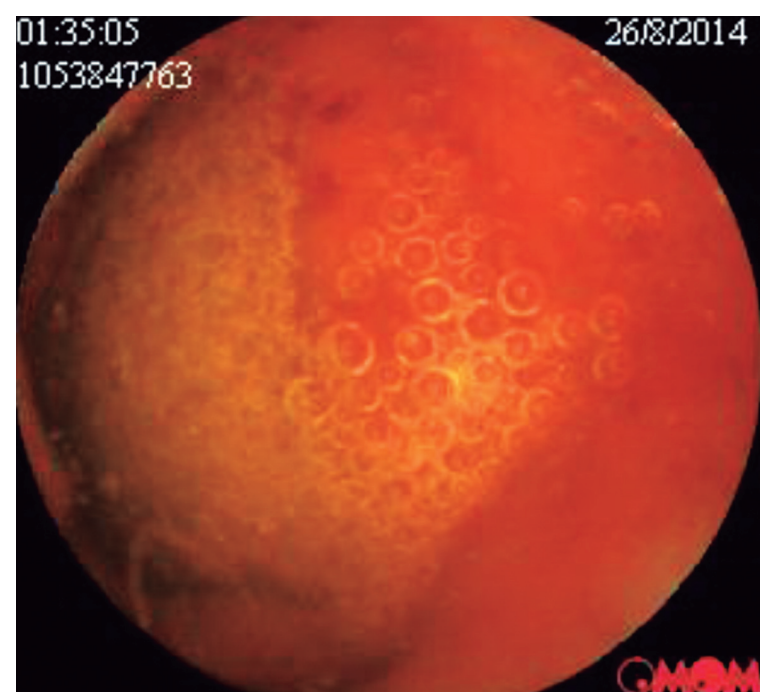

Figura 4. Gran úlcera cubierta de fibrina. Múltiples parásitos adheridos a la pared intestinal. 
en la evaluación de la eficacia de los tratamientos dirigidos a la desparasitación $(12,13)$. Las principales limitaciones de la cápsula endoscópica son la imposibilidad para efectuar medidas terapéuticas y su costo elevado con respecto otras modalidades disponibles. También depende del peristaltismo para su avance, lo que lleva a perder visión de muchas áreas de la superficie mucosa, aunque dicho problema está en resolución en estos momentos, con la disponibilidad de cápsulas que permiten la rotación del lente $360^{\circ}$ para una evaluación completa $(2,14)$.

En relación con el caso presentado, por las características macroscópicas identificadas del parásito, sumado a los hallazgos patológicos locales y las manifestaciones clínicas del paciente, puede hacerse una aproximación diagnóstica a un caso de infestación por $S$. stercoralis, que es un parásito casi transparente, mide entre 2,2 y $2,5 \mathrm{~mm}$, con diámetro de $50 \mu \mathrm{m}$, y vive en túneles cavados entre los enterocitos del intestino delgado del hospedero, similar a lo observado en nuestro caso (15). El examen fecal clásico en busca de parásitos tiene un rendimiento muy bajo para la detección de S. stercoralis, menor al $30 \%$. Por ello, se utilizan frotis y tinciones con lugol o auramina. La recomendación es llevar a cabo un examen periódico de heces para mejorar el rendimiento diagnóstico, aunque existen otras técnicas diagnósticas que, en general, no se hacen de rutina en los laboratorios de los centros que prestan servicios de salud (prueba del embudo, cultivo en placa de agar y método del papel de filtro). El paciente recibió tratamiento con ivermectina en dosis de $1 \mathrm{mg} / \mathrm{kg}$, asociada con albendazol en 200 mg VO, cada 12 horas, durante 10 días, lo que logró la desaparición de los síntomas a la fecha (15).

Existen pocos casos en la literatura de diagnóstico de parasitosis por medio de cápsula endoscópica, pero con el incremento de la utilización de dicho método, en el futuro será más contundente su utilización para este fin $(12,16)$.

\section{REFERENCIAS}

1. Hotez PJ, Molyneux DH, Fenwick A, Kumaresan J, Sachs SE, Sachs JD, et al. Control of neglected tropical diseases. N Engl J Med. 2007;357(10):1018-27. doi: 10.1056/ NEJMra064142.

2. Hotez PJ, Brindley PJ, Bethony JM, King CH, Pearce EJ, Jacobson J. Helminth infections: the great neglected tropical diseases. J Clin Invest. 2008;118(4):1311-21. doi: 10.1172/ JCI34261.

3. Soga K, Handa O, Yamada M, Sakagamia J, Yagi N, Naito $\mathrm{Y}$, et al. In vivo imaging of intestinal helminths by capsule endoscopy. Parasitol Int. 2014;63(1):221-8. doi: 10.1016/j. parint.2013.09.006.

4. Arciniegas E, Corredor A, Hernández CA. Parasitismo intestinal. Bogotá, Colombia: Instituto Nacional de Salud; 2000.
5. Ordóñez LE, Angulo ES. Desnutrición y su relación con parasitismo intestinal en niños de una población de la Amazonia colombiana. Biomedica. 2002;22(4):486-98. doi: 10.7705/biomedica.v22i4.1175.

6. Dupouy-Camet J, Peduzzi R. Current situation of human diphyllobothriasis in Europe. Euro Surveill. 2004;9(5):315. doi: 10.2807/esm.09.05.00467-en.

7. de Marval F, Gottstein B, Weber M, Wicht B. Imported diphyllobothriasis in Switzerland: molecular methods to define a clinical case of Diphyllobothrium infection as Diphyllobothrium dendriticum, August 2010. Euro Surveill. 2013;18(3). pii: 20355.

8. Center for Disease Control and Prevention [Internet]. Diagnosis of Parasitic Diseases. Última actualización: mayo 9 de 2018. Disponible en: http://www.cdc.gov/parasites/ references_resources/diagnosis.html.

9. Ladas SD, Triantafyllou K, Spada C, Riccioni ME, Rey JF, NivY, et al. European Society of Gastrointestinal Endoscopy (ESGE): recommendations (2009) on clinical use of video capsule endoscopy to investigate small-bowel, esophageal and colonic diseases. Endoscopy. 2010;42(3):220-7. doi: 10.1055/s-0029-1243968.

10. Sidhu R, Sanders DS, Morris AJ, McAlindon ME. Guidelines on small bowel enteroscopy and capsule endoscopy in adults. Gut. 2008;57(1):125-36. doi: 10.1136/ gut.2007.129999.

11. Soares J, Lopes L, Villas-Boas G, Pinho C. Ascariasis observed by wireless-capsule endoscopy. Endoscopy. 2003;35(2):194. doi: 10.1055/s-2003-37008.

12. Barnett K, Emder P, Day AS, Selby WS. Tapeworm infestation: a cause of iron deficiency anemia shown by capsule endoscopy. Gastrointest Endosc. 2007;66(3):625-7. doi: 10.1016/j.gie.2006.12.004.

13. Hosoe N, Imaeda H, Okamoto S, Bessho R, Saito R, Ida Y, et al. A case of beef tapeworm (Taenia saginata) infection observed by using video capsule endoscopy and radiography (with videos). Gastrointest Endosc. 2011;74(3):690-1. doi: 10.1016/j.gie.2011.05.011.

14. Awadzi K, Barakat R, Basáñez M, Boatin B, García H, Gazzinelli A, et al. Research Priorities for Helminth Infections. Technical Report of the TDR Disease Reference Group on Helminth Infections. Ginebra, Suiza: World Health Organization; 2012. Disponible en: http:// www.who.int/iris/bitstream/10665/75922/1/WHO TRS 972 eng.pdf.

15. Farthing $\bar{M}$, Fedail S, Savioli L, Bundy D, Krabshuis J. Manejo de la estrongiloidiasis. Milwaukee, Estados Unidos: World Gastroenterology Organisation; 2014. Disponible en: http://www.worldgastroenterology.org/guidelines/ global-guidelines/management-of-strongyloidiasis/management-of-strongyloidiasis-spanish.

16. Hosoe N, Ogata H, Hibi T. Endoscopic imaging of parasites in the human digestive tract. Parasitol Int. 2014;63(1):21620. doi: 10.1016/j.parint.2013.08.003. 